\title{
NRAS Mutation Analysis Was Performed
}

National Cancer Institute

\section{Source}

National Cancer Institute. NRAS Mutation Analysis Was Performed. NCI Thesaurus. Code C160479.

An indication that NRAS mutation analysis was performed during the study. 